\title{
Artefact and ablation performance of an MR-conditional high-power microwave system in bovine livers: an ex vivo study
}

\author{
Antonia Grimm', Moritz Winkelmann', Jakob Weiß', Georg Gohla', Gunnar Blumenstock², Konstantin Nikolaou', \\ Stephan Clasen ${ }^{1}$ and Rüdiger Hoffmann ${ }^{*}$ (D)
}

\begin{abstract}
Background: We evaluated a magnetic resonance (MR)-conditional high-power microwave ablation system. Methods: An ex vivo 1.5-T evaluation was conducted by varying the sequence (T1-weighted volume interpolated breath-hold examination, T1w-VIBE; T1-weighted fast low-angle shot, T1w-FLASH; T2-weighted turbo spin-echo, T2w-TSE), applicator angulation to $B_{0}$ (A-to- $\left.B_{0}\right)$, slice orientation, and encoding direction. Tip location error (TLE) and artefact diameters were measured, and influence of imaging parameters was assessed with analysis of variance and post hoc testing. Twenty-four ex vivo ablations were conducted in three bovine livers at $80 \mathrm{~W}$ and $120 \mathrm{~W}$. Ablation durations were 5, 10, and 15 min. Ablation zones were compared for short-axis diameter (SAD), volume, and sphericity index (SI) with unpaired $t$ test.

Results: The artefact pattern was similar for all sequences. The shaft artefact ( $4.4 \pm 2.9 \mathrm{~mm}$, mean \pm standard deviation) was dependent on the sequence $(p=0.012)$ and the $A$-to- $B_{0}(p<0.001)$; the largest shaft diameter was measured with T1w-FLASH $(6.3 \pm 3.4 \mathrm{~mm})$ and with perpendicular $A$-to- $B_{0}(6.7 \pm 2.4 \mathrm{~mm})$. The tip artefact $(1.6 \pm 0.7 \mathrm{~mm})$ was dependent on $A$-to- $B_{0}(p=0.001)$; TLE was $-2.6 \pm 1.0 \mathrm{~mm}$. Ablation results at the maximum setting (15 min, $120 \mathrm{~W}$ ) were $\mathrm{SAD}=42.0 \pm 1.41 \mathrm{~mm}$; volume $=56.78 \pm 3.08 \mathrm{~cm}^{3}, \mathrm{SI}=0.68 \pm 0.05$. In all ablations, SI ranged $0.68-0.75$ with the smallest SI at $15 \mathrm{~min}$ and $120 \mathrm{~W}(p=0.048)$.
\end{abstract}

Conclusion: The system produced sufficiently large ablation zones and the artefact was appropriate for MR-guided interventions.

Keywords: Ablation techniques, Cattle, Liver, Magnetic resonance imaging, Microwaves

\section{Key points}

- An MR-conditional high-power microwave system was assessed for artefact and ablation performance in bovine ex vivo livers at $1.5 \mathrm{~T}$.

- The applicator artefact is precise concerning tip depiction.

- Largest artefact diameters were measured with a T1-weighted gradient-echo fast low-angle shot sequence.

\footnotetext{
* Correspondence: ruediger.hoffmann@med.uni-tuebingen.de ${ }^{1}$ Department of Diagnostic and Interventional Radiology, University Hospital of Tübingen, Hoppe-Seyler-Strasse 3, 72076 Tübingen, Germany Full list of author information is available at the end of the article
}

- Applicator angulation perpendicular to $B_{0}$ causes largest artefact diameters.

- The MR-conditional microwave system provided appropriate dimensioned ablation zones.

\begin{abstract}
Background
Percutaneous thermoablation is an established minimally invasive treatment option for patients with hepatic malignancies who are not suitable for surgical resection due to impaired hepatic function, comorbidities, or unfavourable anatomic conditions $[1,2]$. With radiofrequency (RF) ablation being the most common representative in the "toolbox" of local therapies, studies have shown that tumour location close to large vessels and size greater
\end{abstract}


than $3 \mathrm{~cm}$ are critical factors regarding local recurrencefree survival [3-5].

Over the last decade, microwaves (MW) were introduced as an energy source and MW ablation (MWA) has developed into an alternative to RF ablation, with theoretical physical advantages and an increasing variety of commercially available MWA systems [6, 7]. Indeed, in comparison to RF ablation, MWA is not limited by desiccation around the applicator, allowing for higher ablation temperatures in larger ablation zones in a shorter time with a single applicator [8, 9]. Furthermore, studies have shown that MWA is less susceptible to the heat-sink effect of larger hepatic vessels [10]. A recent meta-analysis has shown lower local recurrence rates after MWA of larger tumours in comparison to RF ablation [11].

Besides a satisfactory ablation technique, precise applicator placement and therapy monitoring are relevant for successful thermoablation. In this respect, magnetic resonance (MR) as a guidance modality offers several advantages including an assessment of the ablation zone without application of contrast agent, free selection of imaging planes, temperature measurement with $M R$ thermometry and radiation-free near real-time fluoroscopic sequences [12-14]. The high sensitivity of MR imaging (MRI) for small liver lesions is advantageous during tumour targeting and visibility of the smallest target tumours can be increased for hours by administering gadoxetic acid [15-18].

Despite these technical advantages, restricted availability of suitable scanners and higher costs are critical points in view of the relatively long durations of MRguided procedures, so that MRI as guidance modality for thermal ablation is currently limited to specialised centres [19]. In this context, the introduction of an MRconditional high-power MWA system might combine the advantages of both techniques and increase the significance of MR-guided thermoablation in the future by decreasing the procedure duration. A prerequisite for successful MR-guided MWA is a reliable and adequate configuration of the applicator artefact with an accurate depiction of the tip. Large antenna artefacts cause a good visualisation of the applicator, however, may impair the assessment of the ablation zone, so that a balanced artefact configuration is mandatory.

Thus, the purpose of this study was the preclinical evaluation of a new MR-conditional high-power MWA system regarding ablation performance and applicator artefact appearance.

\section{Methods}

Microwave tissue ablation technique and equipment The experiments were conducted with a high-power MWA system with a maximum generator power of $150 \mathrm{~W}$. The system was equipped with a pump for perfusion-cooling of the applicator shaft. The MW generator (ECO-100E2, Nanjing ECO Medical Instrument Co., China) worked at a frequency of $2.45 \mathrm{GHz}$. All experiments were conducted with a 14-G MW applicator (ECO-100AI13C, Nanjing ECO Medical Instrument Co., China) with a shaft length of $15 \mathrm{~cm}$. The applicator is composed of a shaft consisting of titanium alloy and a ceramic tip with a length of $18 \mathrm{~mm}$. A 4-m long coaxial cable connects the antenna with the generator enabling the generator to be positioned safely outside the MR scanner room during ablation.

\section{MRI protocol and artefact evaluation}

Artefact evaluation was conducted in a 1.5 - $\mathrm{T}$ short bore scanner (Magnetom ESPREE, Siemens Healthineers, Erlangen, Germany) with a horizontal main magnetic field $\left(B_{0}\right)$ and a four-channel body-array surface coil. The MW applicator was placed in an MRI phantom consisting of a Plexiglas box filled with a $0.2 \%$ gadolinium solution (Gadovist, Bayer Healthcare, Berlin, Germany). The phantom was positioned at the magnet isocentre and enabled a deflection of the applicator relative to $B_{0}$ between $0^{\circ}$ and $90^{\circ}$. The measurements were performed with three different sequences:

1) A three-dimensional T1-weighted volume interpolated breath-hold examination (T1W-VIBE) with chemically selective fat-saturation pulse, performed with flip angle of $10^{\circ}$, repetition time (TR) of $6.2 \mathrm{~ms}$, echo time (TE) of $1.61 \mathrm{~ms}$, bandwidth of $457 \mathrm{~Hz} /$ pixel, slice thickness of $1 \mathrm{~mm}$, field of view (FOV) $192 \times 192 \mathrm{~mm}$, acquisition matrix $192 \times 192$, and reconstruction matrix $192 \times 192$;

2) A two-dimensional T1-weighted, fast low-angle shot (T1W-FLASH) gradient-echo sequence with periodic chemically selective fat-saturation pulses and flip angle of $70^{\circ}$, TR of $122 \mathrm{~ms}$, TE of $4.36 \mathrm{~ms}$, bandwidth of $139 \mathrm{~Hz} /$ pixel, slice thickness $4 \mathrm{~mm}$, FOV $192 \times 192 \mathrm{~mm}$, acquisition matrix $192 \times 192$, and reconstruction matrix $192 \times 192$;

3) A two-dimensional T2-weighted turbo spin-echo (T2W-TSE) sequence with TR of $3750 \mathrm{~ms}$, TE of $129 \mathrm{~ms}$, flip angle $145^{\circ}$, echo train length 29 , bandwidth $260 \mathrm{~Hz} /$ pixel, slice thickness $4 \mathrm{~mm}$, FOV $192 \times 192 \mathrm{~mm}$, acquisition matrix $192 \times 192$, reconstruction matrix $384 \times 384$.

The following factors were systematically varied: sequence type (T1W-VIBE, T1W-FLASH, T2W-TSE), applicator orientation to $B_{0}\left(0^{\circ}, 45^{\circ}, 90^{\circ}\right)$, slice orientation with respect to the applicator (axial, coronal, sagittal), and encoding direction (phase encoding direction or frequency encoding direction being perpendicular to 
the long axis of the applicator) resulting in a total of 36 artefact measurements. Artefact analysis of the acquired images was performed with the open-source software ImageJ (http://rsb.info.nih.gov/ij). Artefacts were defined according to the American Society for Testing Materials as deviation of $\pm 30 \%$ from the median signal intensity around the applicator [20]. Imaging analyses were conducted in consent of two readers (AG and RH).

The artefact diameters were measured at the applicator tip and the applicator shaft. The tip location error (TLE) was assessed on images with coronal or sagittal orientation in relation to the applicator. The TLE describes the deviation from the measured distance between the distal end of the tip and the Plexiglas model and the actual set $10 \mathrm{~mm}$ distance. A positive TLE correlates with an overestimation of the applicator position in the long axis direction [21].

\section{Ablation protocols and ablation zone evaluation}

All ablations were performed ex vivo at room temperature using three fresh bovine livers (Bos Taurus) obtained from a local abattoir. Before positioning of the MW applicator, large hepatic veins were explored with a metal probe to avoid close positioning. The ablation durations were 5, 10, and 15 min (maximum recommended duration). Ablations were conducted with a power of $80 \mathrm{~W}$ and $120 \mathrm{~W}$ (maximum recommended power for liver ablation with a 14-G antenna). Each combination was repeated four times resulting in a total of 24 ablations. Results were only made available to the manufacturer after completion of the experiments.

After ablation, the antenna was replaced by a metal bar serving as guidance to dissect the liver along the ablation zone. For further measurements, the ablation zone was photographed (Canon, EOS 350D, Tokyo, Japan). The ablation zone diameter along the antenna insertion axis was defined as long-axis diameter (LAD). The largest diameter of the ablation zone perpendicular to the LA was defined as short-axis diameter (SAD) (Fig. 1). Dimensions were determined using callipers by measuring the perimeters of the white coagulation zone. The volume of the ablation zone was calculated using the ellipsoid formula for diameters (Volume $\left.=\pi / 6^{*} \mathrm{LA}^{*}(\mathrm{SAD})^{2}\right)$. The shape of the ablation zone was determined by calculating the sphericity index $(\mathrm{SI})=\mathrm{SAD} / \mathrm{LAD}$.

\section{Statistical analysis}

Acquired data were analysed with the statistical software JMP 13 (SAS Institute, Cary, NC, USA). To compare the TLE, shaft artefact diameter and tip artefact diameter in terms of sequence type, angulation to $B_{0}$, slice orientation and encoding direction analysis of variance (ANOVA) was performed. The assumptions of variance

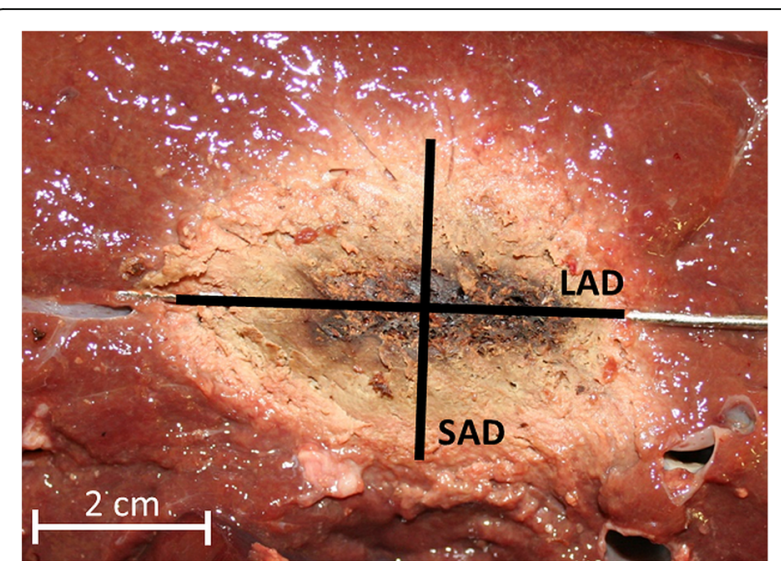

Fig. 1 Cross-section of an ablation zone after 15 min ablation at $120 \mathrm{~W}$. The long axis diameter (LAD) and short-axis diameter (SAD) are shown

homogeneity and normal distribution were checked. In the case of heterogeneity of variances, the Welch ANOVA test was used. If a significant overall effect was found, post hoc between-group comparisons were performed following the closed testing procedure and by using the Student $t$ test [22, 23]. In case of comparing only two parameters, the Student $t$ test was used instead of ANOVA.

To compare the effect of a different ablation power on the SAD, volume, and SI with respect to the ablation duration $(5,10$, and $15 \mathrm{~min})$ unpaired $t$ tests was used. Results were displayed as mean \pm standard deviation (SD). A $p$ value $<0.05$ was considered statistically significant for all tests.

\section{Results}

\section{Applicator artefact}

At all acquired sequences, the applicator presented a similar artefact pattern with a prominent shaft and a smaller tip artefact, distal $18 \mathrm{~mm}$ of the applicator. The appearance of the applicator was homogenous along the shaft and the tip without the appearance of blooming artefacts. Table 1 summarises the measurement results regarding the tip and shaft artefact and the TLE.

The mean diameter of the shaft measured $4.4 \pm 2.9 \mathrm{~mm}$ with significant dependence on the sequence type $(p=0.012)$ and the applicator angulation to $B_{0}(p<0.001$; Fig. 2). The significantly largest shaft artefact was measured with the T1W-FLASH sequence $(6.3 \pm 3.4 \mathrm{~mm}$; Fig. 3). An applicator deflection from $B_{0}$ orientation significantly increased the shaft artefact with a diameter of $1.4 \pm 0.6 \mathrm{~mm}$ at $0^{\circ}$ up to $6.7 \pm 2.4 \mathrm{~mm}$ at $90^{\circ}$ (Fig. 4).

The mean diameter of the applicator tip measured $1.6 \pm 0.7 \mathrm{~mm}$ with statistically significant dependence on the angulation to $B_{0}(p=0.001)$ and the largest diameter 


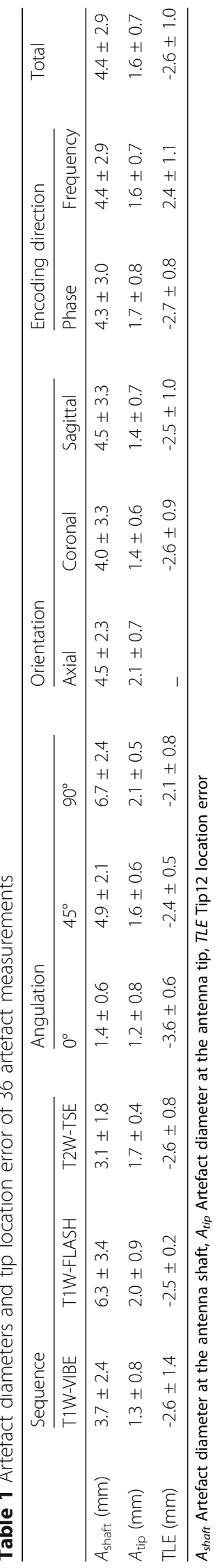




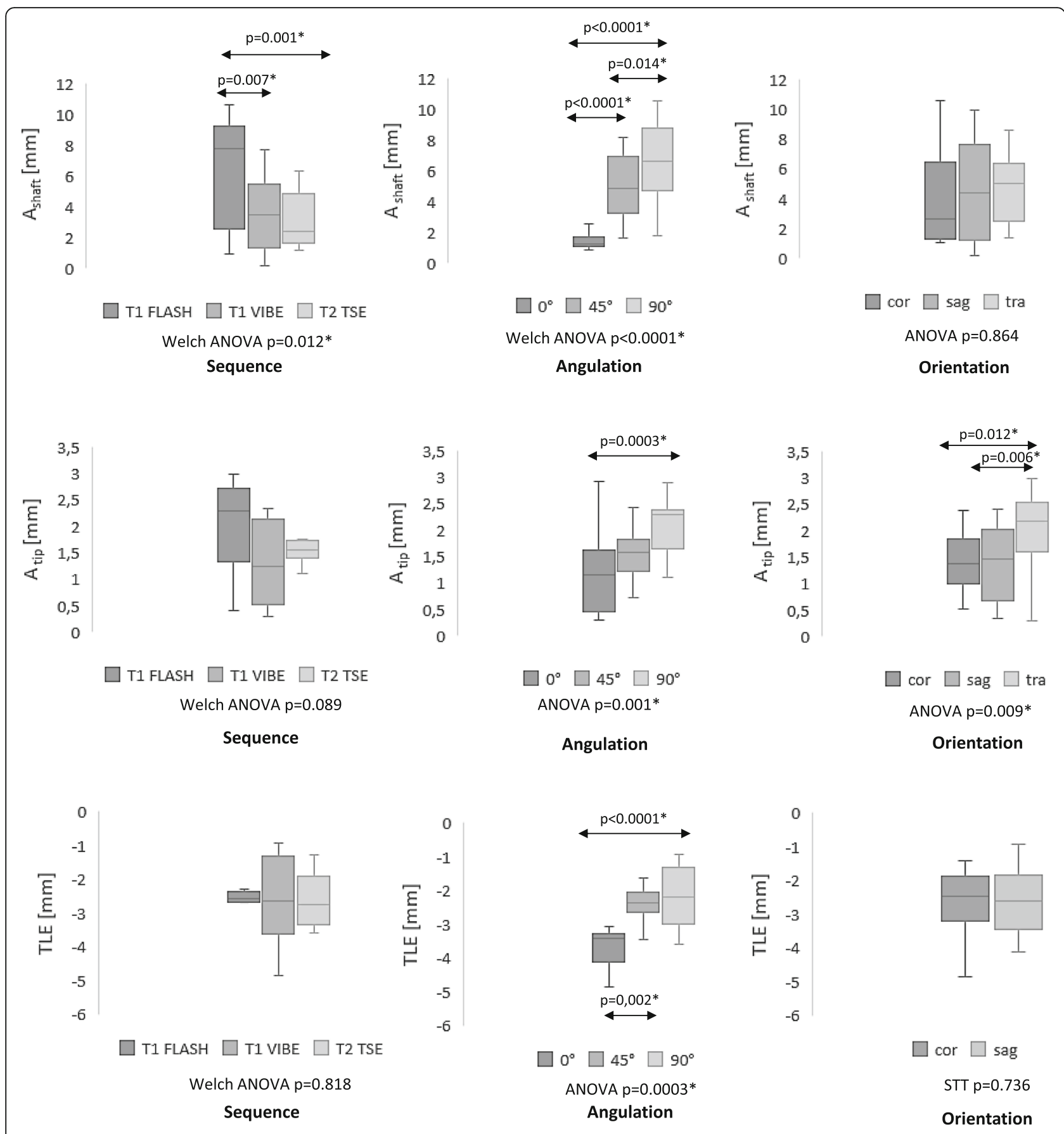

Fig. 2 Statistical analysis regarding the influence of the sequence type, applicator angulation to $B_{0}$ and slice orientation on the applicator artefact. Graphs display: $A_{\text {shaft }}$ (artefact diameter at the antenna shaft), $A_{\text {tip }}$ (artefact diameter at the antenna tip), and TLE (tip location error) of the microwave applicator in relation to sequence type, applicator angulation to $B_{0}$ and slice orientation. Depending on $<2$ or $>2$ parameters Student's $t$ test (STT) or ANOVA was performed. In case of overall statistical significance, the Student $t$ test was performed according to the closed testing procedure. Whiskers indicate the minimum and maximum extreme values; the box indicates the upper and lower quartile. The line in the box indicates the median value

for an applicator angulation perpendicular to $B_{0}$ with a diameter of $2.1 \pm 0.5 \mathrm{~mm}$ (Fig. 4). Largest tip artefact diameters were measured with the T1 FLASH sequence, however, without reaching statistical significance $(p=0.089)$. The largest statistically significant tip diameter was measured for axial slice orientation in relation to the applicator $(p=0.009)$ with a diameter of $2.1 \pm 0.7 \mathrm{~mm}$.

On average, the applicator length was slightly underestimated with a mean TLE of $-2.6 \pm 1.0 \mathrm{~mm}$. The largest 


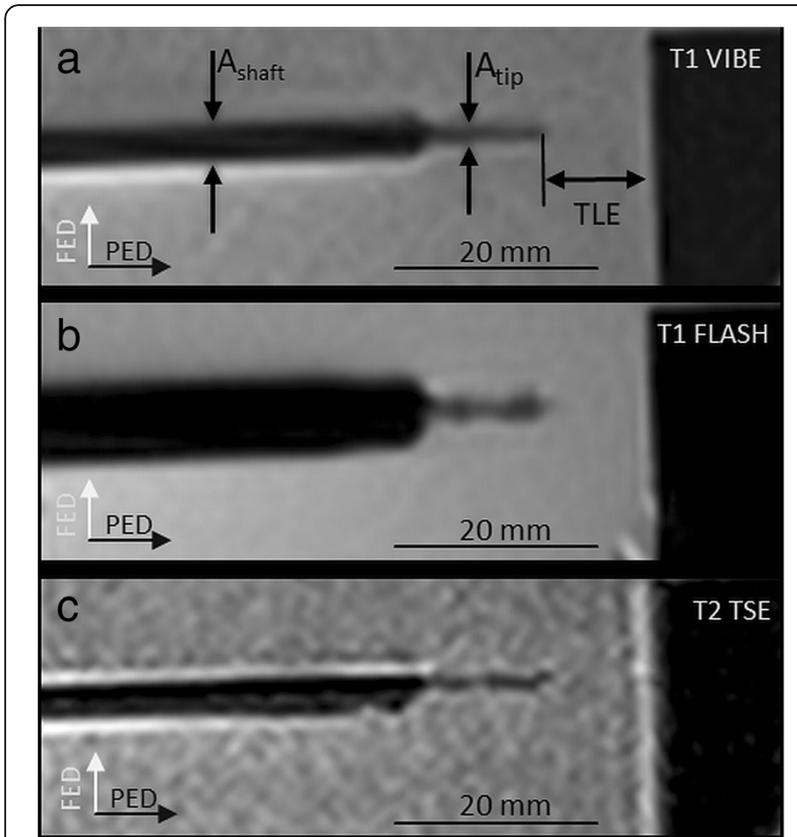

Fig. 3 Artefact of the microwave applicator. Images are acquired under $90^{\circ}$ applicator angulation relative to $B_{0}$ with T1W-VIBE (a) T1W-FLASH (b), and T2W-TSE (c) (see text for sequence details). For all sequences, the applicator shows a similar artefact pattern with a prominent artefact at the shaft $\left(A_{\text {shaft }}\right)$ and a smaller artefact at the tip $\left(A_{\text {tip }}\right)$. PED Phase encoding direction, FED Frequency encoding direction

absolute TLE was measured for an applicator angulation of $0^{\circ}(p<0.001)$ with a TLE of $-3.6 \pm 0.6 \mathrm{~mm}$.

The encoding direction did not affect the artefact diameter of the shaft $(p=0.935)$ and the tip $(p=0.492)$, and the TLE $(p=0.329)$.

\section{Ablation results}

Ablation zone SAD and volume continuously increased with longer ablation durations and were significantly higher with the higher ablation power of $120 \mathrm{~W}$. The highest mean ablation zone volume $\left(56.78 \pm 3.08 \mathrm{~cm}^{3}\right)$ and mean SAD $(42.0 \pm 1.41 \mathrm{~mm})$ was reached using an ablation power of $120 \mathrm{~W}$ and the longest recommended ablation duration $(15 \mathrm{~min})$. The SI ranged between 0.72 and 0.75 at the $5 \mathrm{~min}$ and $10 \mathrm{~min}$ test series, without being significantly affected by the ablation power. With longer ablations $(15 \mathrm{~min})$, the sphericity of the ablation zone significantly decreased with the higher ablation power at $120 \mathrm{~W}(\mathrm{SI}=0.68 \pm 0.05)$ in comparison to ablations at $80 \mathrm{~W}(\mathrm{SI}=0.75 \pm 0.02 ; p=0.048)$.

Table 2 summarises the ablation results regarding $\mathrm{SAD}$, volume, and SI.

\section{Discussion}

Despite the energy transmission via a 4-m coaxial cable, the tested MR-conditional high-power MWA system

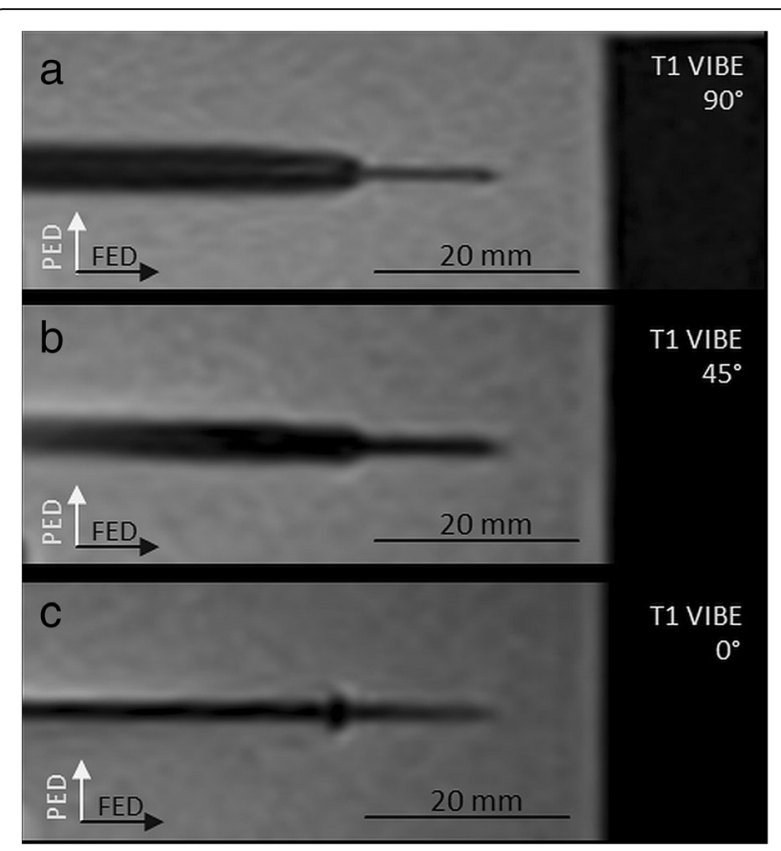

Fig. 4 Artefact of the MW applicator according to the angulation to $B_{0}$. Images are acquired with T1W-VIBE sequence (see text for sequence details) with an applicator angulation to $B_{0}$ of $0^{\circ}(\mathbf{a}), 45^{\circ}(\mathbf{b})$, and $90^{\circ}(\mathbf{c})$. The diameter of shaft artefact was significantly dependent on the angulation with the smallest diameter with an angulation parallel to $B_{0}$. PED Phase encoding direction, FED Frequency encoding direction

reached ablations zones with a SAD above $4 \mathrm{~cm}$ within 15 min, which is comparable to high-power MWA systems designed for computed tomography (CT)-guided procedures [6]. Creation of sufficiently large ablation zones in a short time is relevant, especially to reduce intervention durations for MR-guided procedures. Rempp et al. [24] reported average procedure durations from

Table 2 Comparison of the 24 ablation results at $80 \mathrm{~W}$ and $120 \mathrm{~W}$

\begin{tabular}{llll}
\hline & $80 \mathrm{~W}$ & $120 \mathrm{~W}$ & $p$ value \\
\hline 5 min & & & \\
SAD $(\mathrm{mm})$ & $27.25 \pm 2.63$ & $32.0 \pm 0.82$ & $0.0136^{*}$ \\
Volume $\left(\mathrm{cm}^{3}\right)$ & $14.75 \pm 3.44$ & $23.93 \pm 2.47$ & $0.0049^{*}$ \\
SI & $0.73 \pm 0.08$ & $0.72 \pm 0.02$ & 0.8324 \\
10 min & & & \\
SAD $(\mathrm{mm})$ & $33.5 \pm 1.29$ & $38.0 \pm 1.63$ & $0.0050^{*}$ \\
Volume $\left(\mathrm{cm}^{3}\right)$ & $26.45 \pm 1.76$ & $39.03 \pm 3.69$ & $0.0008^{*}$ \\
SI & $0.75 \pm 0.05$ & $0.74 \pm 0.03$ & 0.7835 \\
15 min & & & \\
SAD (mm) & $38.50 \pm 1.91$ & $42.0 \pm 1.41$ & $0.0259^{*}$ \\
Volume $\left(\mathrm{cm}^{3}\right)$ & $40.15 \pm 5.09$ & $56.78 \pm 3.08$ & $0.0014^{*}$ \\
SI & $0.75 \pm 0.02$ & $0.68 \pm 0.05$ & $0.0480^{*}$ \\
\hline
\end{tabular}

SAD Short axis diameter, $S /$ Sphericity index

*Significant differences 
planning imaging to control imaging of $3.7 \mathrm{~h}$ for MRguided RF ablation in hepatic malignancies with a mean tumour diameter of $20 \mathrm{~mm}$. These procedure durations are a major drawback of MR-guided interventions given the limited availability of MR scanners and higher costs of this modality in comparison to CT and ultrasound $[25,26]$.

The reasons for the long procedure durations in this study were the long net ablation durations of mean $52 \pm 10 \mathrm{~min}$ and the need for applicator repositioning to treat a single tumour (mean $2.4 \pm 1.3$ applicator positions). Up to now, the expectation of shorter procedure durations under MR-guided MWA could not be fulfilled in clinical routine. Hoffmann et al. [27] recently reported procedures durations of $187 \pm 64$ min with a non-cooled, low-power MWA system with a maximum power of $36 \mathrm{~W}$. Similar to the RF ablation study by Rempp et al. [24], multiple applicator repositioning (on average $2.5 \pm 1.2$ ) was necessary to treat the target tumours with a mean diameter of $15 \mathrm{~mm}$. Further studies are necessary to evaluate if the promising ex vivo results of the tested highpower system can be confirmed in vivo, and if the relatively large ablation zones enable a reduction of the number of antenna repositioning procedures in clinical routine so that shorter procedure durations can be achieved.

Besides the volume and the SAD, the sphericity of the ablation zone is another relevant factor for fast and effective tumour ablation [28]. Several studies have shown that most spherical ablation zones were achieved in liver ablations using overlapping ablation zones with multiple antennas or repositioning of a single antenna $[29,30]$. However, placement of multiple antennas or antenna repositioning is time-consuming and may increase the risk of complications due to the need for multiple liver capsule punctures. In our study, the SI ranged from 0.68 to 0.75 . This value is similar to those reported for highpower MWA systems for conventional guidance techniques and higher than the SI value for a non-perfusion cooled MR-conditional system which has been reported to range from 0.36 to 0.59 [31].

Accurate and reliable visualisation of the artefact of the applicator is essential for a fast, safe, and effective tumour ablation. The appearance of the applicator artefact in MRI is predominantly dependent on the material and diameter of the applicator and the difference in magnetic susceptibility between the applicator and the surrounding tissue [32,33]. In our study, the diameter of the applicator shaft artefact was significantly affected by the angulation to the main magnetic field. The largest diameter was measured with a perpendicular angulation to the main magnetic field, which is frequently applied during MR-guided percutaneous procedures in widebore scanners. The prominent artefact diameter at this angulation might be advantageous during applicator positioning. Nevertheless, smaller structures such as small target tumours could be obscured by the artefact, particularly if sequences are acquired which generate larger artefacts such as T1W-FLASH sequence that caused the largest artefacts in our series. However, the measured artefact diameter was smaller than the diameter of MR-conditional RF ablation applicators reported in earlier studies [21, 32]. The applicator tip artefact was clearly smaller than the shaft artefact. In our experimental study with ideal scanning conditions and a high contrast to the surrounding gadolinium solution, this tip area was well visible; however, the small tip artefact might be problematic especially if MR fluoroscopic sequences are used in patients with impaired respiration compliance. On the other hand, a small tip artefact may be beneficial during therapy monitoring. As reported in studies concerning MR-guided RF ablation using applicators with a larger tip artefact, retraction of the applicator can be necessary for evaluation of the target tumour and the ablation zone [24]. Small tip artefacts do not obscure the ablation zone and retraction of the applicator is not necessary for therapy monitoring. Consequently, in cases with an insufficient ablation zone, the ablation can be continued without the need for applicator repositioning [27].

Another essential point for safe applicator positioning is an accurate depiction of the applicator tip. According to the definition of TLE, a negative value implies an underestimation of the applicator length. This is relevant if the target tumour is located in front of critical structures and false depiction of the antenna tip can lead to an accidental puncture of the structure [34]. In our study, the length of the MW applicator was slightly underestimated with a TLE of $-2.6 \pm 1.0 \mathrm{~mm}$. This TLE lays below a TLE of $5 \mathrm{~mm}$, which was considered as a limit for MR-guided musculoskeletal interventions by Wonneberger et al. [35].

The results of our ex vivo study are limited by several factors. First, ex vivo ablations tend to overestimate the size of the ablation zone in comparison to in vivo ablations, due to the absent cooling effect of perfused tissue. On the other hand, we measured the size of the ablation zone after treatment. This zone is smaller than the untreated tissue, as the tissue shrinks during the ablation process, causing an underestimation of the ablated tissue [36]. Our artefact evaluation was conducted in a phantom under optimal conditions with a homogenous and high background signal without moving artefacts such as cardiorespiratory motion. In vivo studies are necessary to confirm the visibility of the device under clinical conditions.

In conclusion, the high-power MR-conditional MWA system tested in this ex vivo study provided a sufficiently dimensioned ablation zone suitable for tumour ablation. 
The artefact of the MW applicator seemed also suitable for MR-guided interventions with an accurate depiction of the applicator tip. These results are encouraging for the application of MR-guided MWA for percutaneous tumour ablation. However, clinical studies are necessary to confirm the potential benefit of the combination of both techniques.

\section{Abbreviations}

FLASH: Fast low-angle shot; FOV: Field of view; LAD: Long axis diameter; MWA: Microwave ablation; RF: Radiofrequency; SAD: Short axis diameter; SI: Sphericity index; TE: Echo time; TLE: Tip location error; TR: Repetition time; TSE: Turbo spin-echo; VIBE: Volume interpolated breath-hold examination

\section{Availability of data and materials}

The datasets used and/or analysed during the current study will be available from the corresponding author on reasonable request.

\section{Funding}

Not applicable

\section{Acknowledgements}

We thank the manufacturer (ECO) for providing the ablation equipment for this study. Results were only made available to the manufacturer after completion of the experiments.

We acknowledge support by Deutsche Forschungsgemeinschaft and Open Access Publishing Fund of University of Tübingen.

\section{Authors' contributions}

AG designed the study. AG, JW, SC, and RH drafted the first version of the entire manuscript. $\mathrm{AG}, \mathrm{RH}$, and $\mathrm{MW}$ conducted the ablation experiments. $A G, R H$, and $G G$ conducted the artefact analysis. GB and AG conducted the statistical analysis. All authors contributed to the interpretation of data, writing, revising, and approving of the final manuscript.

\section{Ethics approval and consent to participate}

Not applicable

\section{Consent for publication}

Not applicable

\section{Competing interests}

The authors declare that they have no competing interests.

\section{Author details}

'Department of Diagnostic and Interventional Radiology, University Hospital of Tübingen, Hoppe-Seyler-Strasse 3, 72076 Tübingen, Germany. ${ }^{2}$ Institute for Clinical Epidemiology and Applied Biometry, University Hospital of Tübingen, Silcherstraße 5, 72076 Tübingen, Germany.

Received: 2 May 2019 Accepted: 24 July 2019

Published online: 23 September 2019

\section{References}

1. Solbiati L, Ahmed M, Cova L, lerace T, Brioschi M, Goldberg SN (2012) Small liver colorectal metastases treated with percutaneous radiofrequency ablation: local response rate and long-term survival with up to 10-year follow-up. Radiology 265:958-968. https://doi.org/10.1148/radiol.12111851

2. Uhlig J, Sellers CM, Stein SM, Kim HS (2019) Radiofrequency ablation versus surgical resection of hepatocellular carcinoma: contemporary treatment trends and outcomes from the United States National Cancer Database. Eur Radiol 29:2679-2689. https://doi.org/10.1007/s00330-018-5902-4

3. Kang TW, Lim HK, Lee MW et al (2015) Aggressive intrasegmental recurrence of hepatocellular carcinoma after radiofrequency ablation: risk factors and clinical significance. Radiology 276:274-285. https://doi.org/1 $0.1148 /$ radiol.15141215

4. Van Cutsem E, Cervantes A, Adam R et al (2016) ESMO consensus guidelines for the management of patients with metastatic colorectal cancer Ann Oncol 27:1386-1422. https://doi.org/10.1093/annonc/mdw235
5. Vahldiek JL, Erxleben C, Bressem KK et al (2018) Multipolar RFA of the liver: Influence of intrahepatic vessels on ablation zones and appropriateness of CECT in detecting ablation dimensions - Results of an in-vivo porcine liver model. Clin Hemorheol Microcirc 70:467-476. https://doi.org/10.3233/ $\mathrm{CH}-189313$

6. Hoffmann R, Rempp H, Erhard L et al (2013) Comparison of four microwave ablation devices: an experimental study in ex vivo bovine liver. Radiology 268:89-97. https://doi.org/10.1148/radiol.13121127

7. Ruiter SJS, Heerink WJ, de Jong KP (2018) Liver microwave ablation: a systematic review of various FDA-approved systems. Eur Radiol 29:40264035. https://doi.org/10.1007/s00330-018-5842-z

8. Fan W, Li X, Zhang L, Jiang H, Zhang J (2012) Comparison of microwave ablation and multipolar radiofrequency ablation in vivo using two internally cooled probes. Am J Roentgenol 198:W46-W50. https://doi.org/10.2214/AJR.11.6707

9. Ryan TP, Brace CL (2017) Interstitial microwave treatment for cancer: historical basis and current techniques in antenna design and performance. Int J Hyperthermia. 33:3-14. https://doi.org/10.1080/02656 736.2016 .1214884

10. Primavesi F, Swierczynski S, Klieser E et al (2018) Thermographic realtime-monitoring of surgical radiofrequency and microwave ablation in a perfused porcine liver model. Oncol Lett 15:2913-2920. https://doi.org/ 10.3892/ol.2017.7634

11. Chinnaratha MA, Chuang MA, Fraser RJ, Woodman RJ, Wigg AJ (2016) Percutaneous thermal ablation for primary hepatocellular carcinoma: a systematic review and meta-analysis. J Gastroenterol Hepatol. 31:294301. https://doi.org/10.1111/jgh.13028

12. Rempp H, Loh H, Hoffmann R et al (2014) Liver lesion conspicuity during real-time MR-guided radiofrequency applicator placement using spoiled gradient echo and balanced steady-state free precession imaging. J Magn Reson Imaging 40:432-429. https://doi.org/10.1002/jmri.24371

13. Winter $L$, Oberacker $\mathrm{E}$, Paul $\mathrm{K}$ et al (2016) Magnetic resonance thermometry: Methodology, pitfalls and practical solutions. Int J Hyperthermia 32:63-75. https://doi.org/10.3109/02656736.2015.1108462

14. Rempp H, Clasen S, Pereira PL (2012) Image-based monitoring of magnetic resonance-guided thermoablative therapies for liver tumors. Cardiovasc Interv Radiol 35:1281-1294. https://doi.org/10.1007/s00270-011-0227-6

15. Rosenberg C, Jahn A, Pickartz T, Wahnschaffe U, Patrzyk M, Hosten N (2014) Gd-EOB-DTPA-enhanced MR guidance in thermal ablation of liver malignancies. PloS One 9:e109217. https://doi.org/10.1371/journal.pone. 0109217

16. Weiss J, Hoffmann R, Rempp H et al (2019) Feasibility, efficacy, and safety of percutaneous MR-guided ablation of small $(\leq 12 \mathrm{~mm})$ hepatic malignancies. J Magn Reson Imaging 49:374-381. https://doi.org/10.1002/jmri.26252

17. Terraz S, Cernicanu A, Lepetit-Coiffé M et al (2010) Radiofrequency ablation of small liver malignancies under magnetic resonance guidance: progress in targeting and preliminary observations with temperature monitoring. Eur Radiol 20:886-897. https://doi.org/10.1007/s00330-009-1611-3

18. Jhaveri KS, Fischer SE, Hosseini-Nik $\mathrm{H}$ et al (2017) Prospective comparison of gadoxetic acid-enhanced liver MRI and contrast-enhanced $C T$ with histopathological correlation for preoperative detection of colorectal liver metastases following chemotherapy and potential impact on surgical plan. HPB (Oxford) 19:992-1000. https://doi.org/10.1016/j.hpb.2017.06.014

19. Hoffmann R, Thomas C, Rempp H et al (2012) Performing MR-guided biopsies in clinical routine: factors that influence accuracy and procedure time. Eur Radiol 22:663-671. https://doi.org/10.1007/s00330-011-2297-x

20. American Society for Testing and Materials (2013) F2119-07: Standard test method for evaluation of MR image artifacts from passive implants, ASTM International, West Conshocken. Available via www.astm.org

21. Hoffmann R, Rempp H, Eibofner $F$ et al (2016) In vitro artefact assessment of a new MR-compatible microwave antenna and a standard MR-compatible radiofrequency ablation electrode for tumour ablation. Eur Radiol 26:771-779. https://doi.org/10.1007/s00330-015-3891-0

22. Bauer P (1991) Multiple testing in clinical trials. Stat Med 10:871-889. https://doi.org/10.1002/sim.4780100609

23. Bender R, Lange S (2001) Adjusting for multiple testing - when and how? J Clin Epidemiol 54:343-349. https://doi.org/10.1016/S0895-4356(00)00314-0

24. Rempp H, Waibel L, Hoffmann R, Claussen CD, Pereira PL, Clasen S (2012) MR-guided radiofrequency ablation using a wide-bore 1.5-T MR system: clinical results of 213 treated liver lesions. Eur Radiol 22:1972-1982. https://doi.org/10.1007/s00330-012-2438-x 
25. Maurer MH, Froeling V, Röttgen R et al (2014) MRl-guided and CT-guided cervical nerve root infiltration therapy: a cost comparison. Rofo 186:559-566. https://doi.org/10.1055/s-0033-1356169

26. D'Onofrio M, Beleù A, De Robertis R (2019) Ultrasound-guided percutaneous procedures in pancreatic diseases: new techniques and applications. Eur Radiol Exp 3:2. https://doi.org/10.1186/s41747-018-0081-2

27. Hoffmann R, Rempp H, Keßler DE et al (2017) MR-guided microwave ablation in hepatic tumours: initial results in clinical routine. Eur Radiol 27:1467-1476. https://doi.org/10.1007/s00330-016-4517-x

28. Vogl TJ, Basten LM, Nour-Eldin NA et al (2018) Evaluation of microwave ablation of liver malignancy with enabled constant spatial energy control to achieve a predictable spherical ablation zone. Int J Hyperthermia 34:492-500. https://doi.org/10.1080/02656736.2017.1358408

29. Harari CM, Magagna M, Bedoya M, et al (2016) Microwave ablation: comparison of simultaneous and sequential activation of multiple antennas in liver model systems. Radiology 278:95-103. https://doi.org/10.1148/radiol.2015142151

30. Brace CL, Sampson LA, Hinshaw JL, Sandhu N, Lee FT Jr (2009) Radiofrequency ablation: simultaneous application of multiple electrodes via switching creates larger, more confluent ablations than sequential application in a large animal model. J Vasc Interv Radiol 20:118-124. https://doi.org/10.1016/j.jvir.2008.09.021

31. Hoffmann R, Kessler DE, Weiss J et al (2017) Preclinical evaluation of an MR-compatible microwave ablation system and comparison with a standard microwave ablation system in an ex vivo bovine liver model. Int J Hyperthermia 33:617-623. https://doi.org/10.1080/02656736.2017.1284349

32. Aube C, Schmidt D, Brieger J et al (2004) Magnetic resonance imaging characteristics of six radiofrequency electrodes in a phantom study. J Vasc Interv Radiol 15:385-392. https://doi.org/10.1097/01.RVI.0000121408.46920.F1

33. Liu H, Hall WA, Martin AJ, Truwit CL (2001) Biopsy needle tip artifact in MR-guided neurosurgery. J Magn Reson Imaging 13:16-22 https://doi.org/ 10.1002/1522-2586(200101)13:1<16::AID-JMRI1003>3.0.CO;2-B

34. Thomas C, Springer F, Rothke M et al (2010) In vitro assessment of needle artifacts with an interactive three-dimensional MR fluoroscopy system. J Vasc Interv Radiol 21:375-380. https://doi.org/10.1016/j.jvir.2009.11.012

35. Wonneberger U, Schnackenburg B, Streitparth F, Walter T, Rump J, Teichgräber UK (2010) Evaluation of magnetic resonance imaging-compatible needles and interactive sequences for musculoskeletal interventions using an open high-field magnetic resonance imaging scanner. Cardiovasc Intervent Radiol 33:346-351. https://doi.org/10.1007/s00270-009-9676-6

36. Farina L, Nissenbaum Y, Cavagnaro M, Goldberg SN (2018) Tissue shrinkage in microwave thermal ablation: comparison of three commercial devices. Int J Hyperthermia 34:382-391. https://doi.org/10.1080/02656736.2017. 1362115

\section{Publisher's Note}

Springer Nature remains neutral with regard to jurisdictional claims in published maps and institutional affiliations.

\section{Submit your manuscript to a SpringerOpen ${ }^{\circ}$ journal and benefit from:}

- Convenient online submission

- Rigorous peer review

- Open access: articles freely available online

- High visibility within the field

- Retaining the copyright to your article

Submit your next manuscript at $\boldsymbol{\nabla}$ springeropen.com 